\title{
Application Analysis of Binocular Vision Technology in Intelligent Robot
}

\author{
Lijuan Zou ${ }^{1}$, Mei Lin ${ }^{1}$, Hongqiang Guo ${ }^{1 *}$ \\ ${ }^{1}$ College of mechanical and electrical engineering, Binzhou City, Shandong Province, 256600 \\ *bgzoulijuan@edu.cn
}

\begin{abstract}
At present, under the background of the continuous improvement of the level of industrial automation, the application of intelligent robots is becoming more and more common. In order to give full play to the function of intelligent robot, it is necessary to strengthen the effective application of binocular vision technology in intelligent robot. In the specific design process of intelligent robot, it is necessary to understand the working principle and application advantages of binocular vision technology, and grasp the specific research status of the current binocular vision robot. On this basis, the key technology of binocular vision technology in intelligent robot is analyzed to improve the design level of intelligent robot.
\end{abstract}

Keywords: binocular vision technology, Intelligent robot, Application of the point.

\section{PREFACE}

With the continuous development of robot technology, the application of robots in different fields can make up for the problem of labor shortage in the stage of rapid economic development. In the stable period of China's social economy, the industrial structure has produced new changes, the application of robots is more and more common. In addition to operating in some harsh environments, robots can also replace manual work to complete some relatively large harm to the human body. In the process of intelligent robot research and development, it is very important to fully apply binocular vision technology. Binocular vision technology can enable the robot to comprehensively analyze the threedimensional environment and distinguish the specific shape, position and movement of the object in the threedimensional environment. At the same time, it can describe, identify, store and understand relevant information. Binocular vision technology is an important sensing technology in the process of passive measurement in computer application. Based on the related technology of human vision processing, the robot can use this technology to simulate human vision. In the process of computer vision method research, it is very important to study and analyze binocular vision technology deeply. It is one of the main research topics of artificial intelligence technology, and also an important basis to promote the development of robot technology in the future.

\section{OVERVIEW OF BINOCULAR VISION TECHNOLOGY}

\subsection{Principles of binocular vision technology}

In the application of intelligent robot, it is necessary to understand the working principle of binocular vision technology. The three-dimensional measurement of binocular vision technology is a simple binocular vision system based on vision principles. The construction principle is shown in Figure 1. In the application process of binocular vision technology, it is necessary to develop artificial eyes similar to human eyes based on human visual system, which is the main visual system of robot. Binocular stereo vision is an important topic in robot vision research. This visual technology is mainly based on the human binocular visual system to the distance perception method. Binocular stereo vision can perceive three-dimensional information. In practical application, the image information of two or more cameras on the same thing at different positions should be obtained by triangulation method, and the distance information should be recovered mainly by visual difference [1]. 


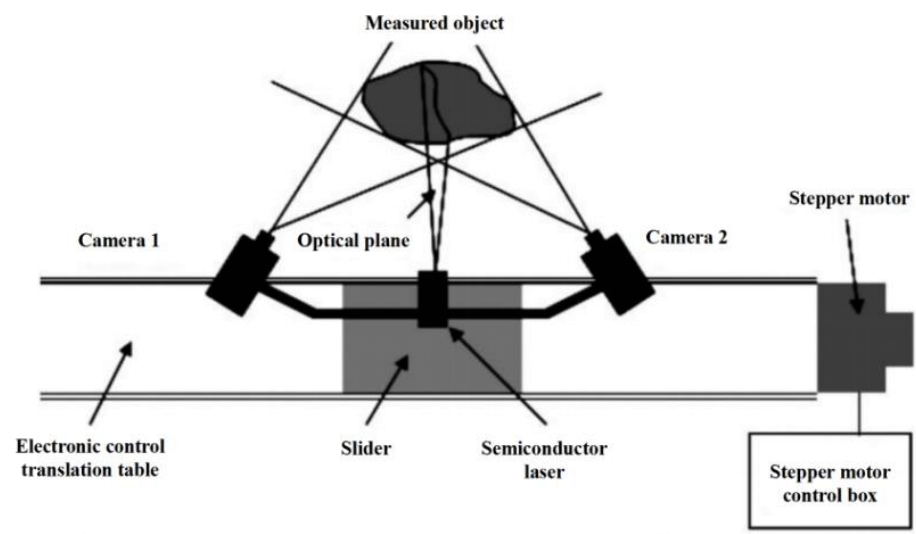

Fig. 1 Principle of binocular vision system

In the process of specific research, based on the relatively simple ideal binocular vision system, the specific application skills of binocular vision technology in intelligent robot are analyzed. In the construction process of the system, two cameras need to be placed parallel to different positions, the center of the image is the optical center of the camera, so as to obtain different images of the same object. This allows a relatively simple binocular vision imaging model to be drawn, as shown in Figure 2. During the operation of the system, it is assumed that the two cameras are identical. On the same plane, $\mathrm{Cl}$ and $\mathrm{CR}$ are the optical centers of the left and right cameras, which is also the origin of the coordinate system. XL and XR are on the same line, and the corresponding $\mathrm{Y}$ and $\mathrm{Z}$ axes of the two cameras are parallel to each other. At this point, the distance between the origin $\mathrm{CL}$ and $\mathrm{CR}$ of the two cameras is $\mathrm{B}$, the origin of the imaging plane is OL and OR respectively, and the distance between the camera and the imaging position is the focal length. When the spatial point $P$ is connected to $\mathrm{OL}$, the intersection point of the imaging plane is $\mathrm{Pl}$; when the spatial point $\mathrm{P}$ is connected to $\mathrm{OR}$, the intersection point of the imaging plane is Pr. This ideal structure model can quickly find the coordinate position of PL and PR. However, due to the influence of various factors in the practical application, the camera placement is difficult to reach the ideal state. In order to allow the two cameras to view a larger common area, the distance between the cameras must be reduced, and the decrease of the baseline distance B will have a certain impact on the accuracy of the data. Therefore, in the application of binocular vision technology in the intelligent robot, it is necessary to carry out in-depth exploration.

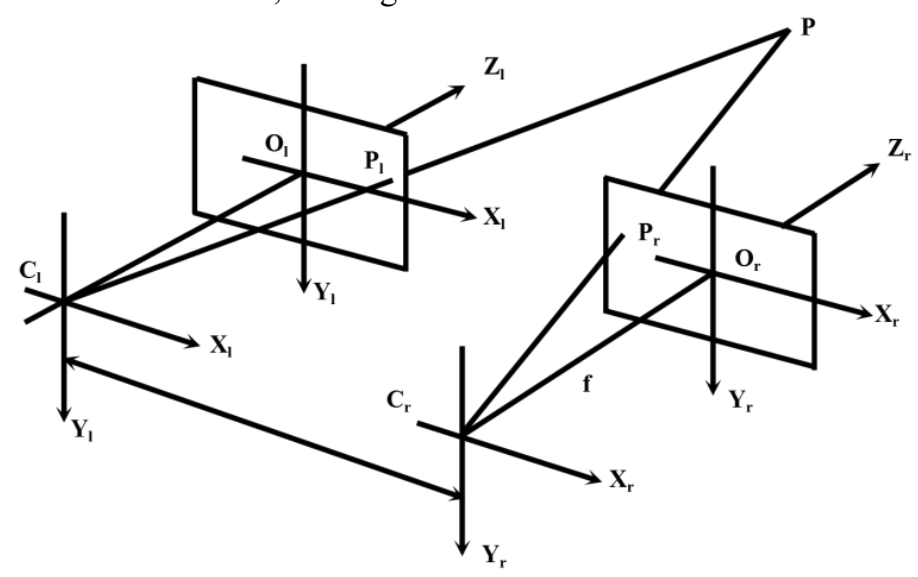

Fig. 2 Schematic diagram of binocular vision imaging principle

\subsection{Application advantages of binocular vision technology}

In the process of studying binocular vision technology, it is necessary to ensure that the technology can effectively obtain the specific information of threedimensional scene in the practical application. At present, there are two ways for computer vision system to obtain relevant information of $3 \mathrm{D}$ scene, including passive vision and active vision. First, passive vision. This kind of vision technology does not need the use of special light source, can directly use the camera to obtain the object image information, and then use the computer to complete the image processing operation, in the visual error can restore the three-dimensional information. Passive vision includes monocular vision and multiocular vision. Monocular vision refers to only use a camera to complete the object shape perception, and eye vision is in general more ambient light or natural light conditions, the use of a certain distance of the two cameras to obtain the object image, then the space object 
point corresponding points in two images, so that we can get the specific distance information. Second, active vision. Active vision mainly obtains the distance information of the object by photoelectric sensing method. There are many specific methods of active vision, such as flying point time method, Mohr technique method, etc. The key content of active vision technology in the application process is that there must be special light source, and at the same time, the unit position and brightness must be strictly controlled, otherwise it will have a negative effect on the result of object information acquisition.

In the process of the application of binocular vision technology by intelligent robot, different data modes can be reasonably selected according to the specific application requirements, and there are certain differences in the applicable scope of different visual modes. Among them, the measurement accuracy of active vision is relatively high, but it has certain requirements for the light source. Therefore, the application cost of the equipment is relatively high, and the scope of application is relatively small. The main function of monocular vision in passive vision is to restore the orientation of the three-dimensional object surface. In order to obtain the three-dimensional structure of the object, multiple measurements are needed from different directions, which may lead to the increase of measurement error. Therefore, in the current intelligent robot research and development process, the application of binocular vision technology has outstanding advantages, mainly manifested in the following aspects: First, the application cost of binocular vision technology is relatively low. In the design process of binocular vision system of intelligent robot, two cameras are used as the equipment. There is no need to use special equipment to obtain image information, and the requirement of light source is relatively low, which can further improve the measurement effect of binocular vision. Second, in the process of binocular vision technology measurement, the results of the accuracy is relatively high. The absolute error of the calculation results of binocular vision system is about $0.3 \mathrm{~mm}$, which can meet the precise requirements of intelligent robot to measure the relevant object information. Thirdly, in the application process of binocular vision technology, the requirement for light source is not high. It only needs to use the camera to obtain the relevant information of $3 \mathrm{D}$ objects on the basis of natural light or ambient light, and the application scope is relatively wide [2].

\section{DEVELOPMENT STATUS OF BINOCULAR VISION INTELLIGENT ROBOT}

In recent years, binocular vision technology in the intelligent robot in Europe and the United States and other developed countries research time is relatively mature, especially with the continuous development of computer technology, optoelectronics technology, some more developed countries have launched a relatively high level of intelligent robots. Compared with developed countries, the research of binocular vision robot in China started relatively late, and the research of binocular vision technology is still in the early stage of development. But some universities and research institutions have also made some achievements. At present, many universities and research institutions in China have begun to conduct in-depth research on binocular vision technology. For example, the Mars 863 project research group has made full use of the threebinocular vision technology, which can complete the accurate measurement of the three-dimensional size of the human body. The measurement speed is fast, and the accuracy of the measurement results is relatively high. Some colleges and universities use binocular vision technology, can also complete the dynamic position detection of multi-degree of freedom mechanical devices; Some colleges and universities can use binocular vision technology for non-contact measurement of irregular objects in three-dimensional space, and the measurement accuracy is relatively high. And some universities also have in-depth research on the specific application of binocular vision technology in intelligent robots. For example, Harbin Institute of Technology can complete the motion navigation design of autonomous soccer robot by using heterogeneous binocular vision technology in the research, and the navigation quality is good. In order to further promote the application and promotion of binocular vision technology in intelligent robots, relevant theories and implementation methods of binocular vision system need to be further explored to improve the practicability of binocular vision technology [3].

\section{KEY POINTS OF APPLICATION OF BINOCULAR VISION TECHNOLOGY IN INTELLIGENT ROBOT}

At present, in the application of binocular vision system, it is necessary to analyze the key technologies of intelligent robot in the application. To ensure that binocular vision technology can be effectively applied in intelligent robot to form a complete binocular stereo vision system. In general, you need to master the following key skills:

\subsection{Image Acquisition}

Image acquisition technology is an important technology in the application process of binocular vision system, and it is also the most basic technology type. When the stereo image is acquired, the 2D image of the $3 \mathrm{D}$ object is mainly taken by the camera, which is the basic condition in stereo vision. The quality of the image must be guaranteed in the process of image acquisition. Therefore, it is necessary to fully grasp the various factors that affect image quality. In general, the shooting 
position of the camera, the geometric features of the camera and the lighting conditions during the shooting process will have a certain impact on the image quality. In the process of specific image acquisition, it is necessary to meet the specific application requirements of the camera according to the actual situation. At the same time, various factors such as viewpoint difference, camera performance, lighting and environmental conditions during the shooting process must be considered. Only in this way can accurate and reasonable calculation be carried out to ensure the accuracy of the measurement results.

\subsection{Calibration camera}

Accurate camera calibration is also the key technology in the application of binocular vision technology. The accurate position of 3D object and corresponding geometric information can be obtained by using the image information of camera. In order to accurately obtain the relationship between spatial points and image pixel points, the accuracy of camera calibration must be guaranteed. Using camera calibration can establish an effective camera forming model, which can determine the internal and external attribute parameters of the camera, so as to obtain the corresponding relationship between spatial points and pixel points, and ensure the accuracy and rationality of relevant data information. In the development of binocular vision system, when two cameras are calibrated, the internal and external parameters of the two cameras are generally obtained by the single camera calibration method, and then the position relationship of the two cameras is established by a set of fixed marks of the same world coordinate. Commonly used single camera calibration methods mainly include the following: First, in the camera calibration process, the traditional photogrammetric calibration method needs to use at least 17 parameters to describe the ending relationship between the camera and the $3 \mathrm{D}$ object controls, which requires a large amount of calculation. The use of binocular vision system technology is not widespread. Second, direct linear transformation. In the application process, the relevant parameters are relatively few, and the calculation efficiency and accuracy are relatively high [4]. Third, perspective transformation matrix method. This computational camera calibration method mainly constructs the camera imaging model through perspective transformation, does not need the initial value, can be calculated in real time, and has positive help to improve the computational efficiency. Fourthly, the camera calibration two-step method. In the application of this method, the camera parameters of the linear system should be obtained by using the perspective matrix transformation method, and then the obtained parameters should be taken as the initial values, the distortion factors should be considered comprehensively, and the nonlinear solution should be obtained by using the optimal method, so as to achieve the purpose of camera calibration. This method will greatly improve the accuracy of camera calibration. Fifthly, biplanar calibration method. In the process of binocular camera calibration, the accuracy of external parameters is relatively high. However, because the accuracy of the structure configuration is relatively low, the distance and perspective of the two cameras will be limited to some extent. Usually, it is necessary to take at least 6 or more known world coordinate points in the application process to obtain a relatively ideal parameter matrix. Therefore, the corresponding process in the actual measurement is relatively complex, and the effect is not always able to reach the expected, which will limit its application scope. In addition, in the process of binocular camera calibration, we must also consider the measurement range of lens nonlinear correction and the corresponding accuracy. Therefore, this method is not universal.

\subsection{Extraction of image features}

At present, in the application of binocular vision technology in intelligent robot, it is necessary to extract appropriate image features, so as to effectively match the corresponding relationship between the images taken by the same object in the same scene in two different positions. At present, there is no universally applicable theory in the process of image feature extraction, which may lead to the diversification of matching features. In general, the matching features in the process of feature extraction mainly include point-like features, linear features and regional features. The image information of large-scale features is more abundant and the amount of data is relatively large, so the matching difficulty is relatively low. However, the accuracy of image information extraction is relatively poor, and it is difficult to describe and extract the corresponding image features. In the process of image information processing of small scale features, the corresponding information amount is relatively small, and the data is relatively large. Therefore, it is necessary to have a strong constraint criterion and matching strategy to ensure the accuracy and effectiveness of matching, overcome the ambiguity matching, and improve the computational efficiency to ensure the reliability of feature extraction. In the detection of matching features, it is necessary to ensure that the matching features have the ability of distinguishing, stability, invariance, uniqueness and the ability to effectively solve the ambiguity matching.

\subsection{Stereo matching}

Stereo matching is to calculate the selected image features, establish the corresponding relationship between the features, find out the mapping points of the same space physical dot in different images, and then obtain the parallax image. This is a difficult problem in the implementation of binocular vision technology. In the 
process of specific application, need the $3 \mathrm{~d}$ objects within the space projection for a $2 \mathrm{~d}$ image, under the different point of view, the image of the same object change is bigger, geometric characteristics, noise and the objects in the scene and the influence of various factors such as light conditions, will to some extent, resulting in a decline in the accuracy of the calculation results. Therefore, various interference factors must be comprehensively considered in the stereo matching process, and effective measures should be taken to eliminate the interference factors, so as to improve the accuracy and reliability of the calculation results as far as possible. At present, the more commonly used matching algorithms include the following :(1) region matching. In practical application, the information accuracy can be improved in places with relatively gentle changes and rich details mainly through the correlation degree of gray information between local Windows. However, it is difficult to select the matching window size of this algorithm, and it is necessary to use window shape technology to match the position with discontinuous parallax, and the calculation is also large. (2) The feature matching does not depend on gray level information, and has strong anti-interference performance, relatively small computation amount and relatively fast computation speed. However, due to the sparsity of the image in the process of feature matching, only sparse parallax fields can be obtained by feature matching. (3) Phase matching. Phase matching is a matching algorithm developed and applied in recent years. As the basic matching unit, phase matching reflects the structural information of the signal and can effectively suppress the high-frequency noise of the image. It is suitable for parallel processing and can obtain the compact parallax with sub-pixel precision. But in the application of phase matching there are some problems such as phase singularity and phase winding which can be solved by using adaptive filter.

\subsection{Deep Recovery}

The comfort distance must be recovered effectively after obtaining the stereo model and matching parallax. In general, the factors that have negative effects on the measured distance mainly include matching positioning error, camera calibration error and feature detection results. In order to ensure the validity and reliability of the measured distance, it is necessary to make comprehensive analysis of different factors to obtain accurate distance recovery. After obtaining the distance information, the existing errors should be corrected. During the acquisition of measurement data, some errors may be caused by many factors. In this case, it is necessary to find out the actual causes and methods of the errors according to the specific manifestations of the errors, and take scientific and reasonable measures to correct the errors, so as to ensure the accuracy of the measurement results [5].

\section{CONCLUSION}

In a word, in the process of intelligent robot research, binocular vision technology must be effectively applied to improve the intelligence and automation of the robot. At present, our country the study of binocular vision technology is still in its early stages, in order to give full play to the application of the binocular vision technology advantage, we must strengthen the innovation of the binocular vision technology research and in-depth discussion, will be able to master the binocular vision technology to deal with the difficulty in the process of application, and take effective measures to solve the problem, promote the application of the binocular vision technology level. In the process of continuous development and improvement of various advanced technologies, the application prospect of binocular vision technology in the future is very broad, and it has important practical significance to promote the further development of intelligent robot research and development in China.

\section{REFERENCES:}

[1] Weng Hewang. Research on the Application of Binocular Vision Technology in Intelligent Robot [J]. Journal of Chifeng University (Natural Science Edition), 2016, 32(021):22-24.

[2] Zhou Zhongwei. Research on Visual Guiding Technology of Intelligent Grasp Robot [D]. Jiangxi University of Science and Technology, 2015.

[3] Yu Jun. Research on Robot Target Detection and Control Based on Binocular Vision [D]. Beijing Jiaotong University.

[4] Dong Hao. Research on Robot Flexible Sorting System Based on Binocular Vision Technology [D]. Shijiazhuang Tiedao University, 2019.

[5] Xue Wenkai. Research on Machine Vision Technology in Assembly Line [D]. Zhengzhou University, 2018. 